\title{
Glassy dynamics of crystallite formation: The role of covalent bonds
}

\author{
Robert S. Hoy ${ }^{a, b, *}$ and Corey S. O'Hern ${ }^{a, b}$
}

\author{
Received Xth XXXXXXXXXX 20XX, Accepted Xth XXXXXXXXX 20XX \\ First published on the web Xth $X X X X X X X X X X 200 X$ \\ DOI: 10.1039/b000000x
}

\begin{abstract}
We examine nonequilibrium features of collapse behavior in model polymers with competing crystallization and glass transitions 'using extensive molecular dynamics simulations. By comparing to "colloidal" systems with no covalent bonds but the same non-bonded interactions, we find three principal results: (i) Tangent-sphere polymers and colloids, in the equilibrium-crystallite phase, have nearly identical static properties when the temperature $T$ is scaled by the crystallization temperature $T_{\text {cryst }}$; (ii) Qualitative features of nonequilibrium relaxation below $T_{\text {cryst }}$, measured by the evolution of local structural properties (such as 'the number of contacts) toward equilibrium crystallites, are the same for polymers and colloids; and (iii) Significant quantitative differences in rearrangements in polymeric and colloidal crystallites, in both far-from equilibrium and near-equilibrium systems, 'can be understood in terms of chain connectivity. These results have important implications for understanding slow relaxation processes in collapsed polymers, partially folded, misfolded, and intrinsically disordered proteins.
\end{abstract}

\section{Introduction}

Collapse transitions of single chain polymers induced by 'changing control parameters such as temperature or solvent quality yield rich nonequilibrium behavior when the rate at 'which these control parameters are changed exceeds characteristic (slow) dynamical rates. Investigating the glassy dynamics of polymer collapse is important for understanding e.g. crystallization kinetics and protein misfolding, yet the majority of studies have focused on equilibrium behavior. In this manuscript, we characterize the nonequilibrium and near'equilibrium collapse and crystallization dynamics of single flexible polymer chains.

We employ a minimal model that yields competing crystallization and glass transitions. Monomers are modeled as monodisperse tangent spheres with hard-core-like repulsive and short-range attractive interactions. Recent studies ${ }^{1,2}$ have shown that in equilibrium, model polymers with narrow square-well interactions exhibit direct "all-or-nothing" crystallization transitions that mimic the discrete folding transition observed in experimental studies of proteins. $\frac{3}{3}$ Short-range attractions also give rise to degenerate, competing ground states, which kinetically hinder collapse to equilibrium crystallites. The associated rugged energy landscapes are believed to control the behavior of intrinsically disordered proteins. $-\underline{4-6}$

A novel aspect of our work is quantitative comparison of polymer collapse dynamics to that of 'colloidal' systems with the same secondary interactions but no covalent bonds. Poly-

${ }^{a}$ Department of Mechanical Engineering and Materials Science, Yale University, New Haven, CT, USA 06520-8286

${ }^{b}$ Department of Physics, Yale University, New Haven, CT 06520-8120

*Email: robert.hoy@yale.edu mers are distinguished from other systems by their topology; connectivity and uncrossability constraints imposed by covalent backbone bonds give rise to cooperative dynamics $\underline{ }$ and phase transitions $\frac{8}{2}$ not present in nonpolymeric materials. Our choice of tangent monodisperse spheres yields identical lowenergy states for polymers and colloids, but (because of the covalent backbone) very different free energy landscapes. $\underline{9}$ This greatly facilitates a robust comparison of crystallite formation and growth dynamics that isolates the role of topology and allows us to isolate and quantify the contributions of the covalent bonds and chain uncrossability to cooperative rearrangements and slow dynamics during collapse. We perform this comparison using extensive molecular dynamics simulations of thermal-quench-rate-dependent collapse and post-quench growth of polymeric and colloidal crystallites.

Figure 1 depicts rate-dependent collapse behavior of systems interacting via hard-core-like repulsions and short-range attractions. $\underline{11}$ In the limit of slow quench rates $|\dot{T}|<\left|\dot{T}^{*}\right|$, where $\dot{T}^{*}$ is a critical quench rate, finite systems exhibit a first-order-like transition from a high-temperature "gas" (for polymers, a self-avoiding random coil) phase to crystallites. The equilibrium transition occurs if $|\dot{T}|$ is small compared to key relaxation rates, such as the crystal nucleation rate $r$ and rate $s$ of large rearrangements in compact structures. At larger $|\dot{T}|$, systems fall out of equilibrium, and pass onto the metastable liquid branch. If $T$ becomes low enough such that $s(T) \gg|\dot{T}|$, the systems become glassy and disorder is frozen in at $T=T_{\text {glass }}$. Otherwise, systems relax towards equilibrium crystallites, as indicated by the downward arrow.

Our analyses compare collapse behavior within the framework of Fig. 1 We find that polymers and colloids behave similarly in many ways, and differences can be linked directly

This journal is @ The Royal Society of Chemistry [year] Journal Name, 2010, [vol]، 1-13 |1 


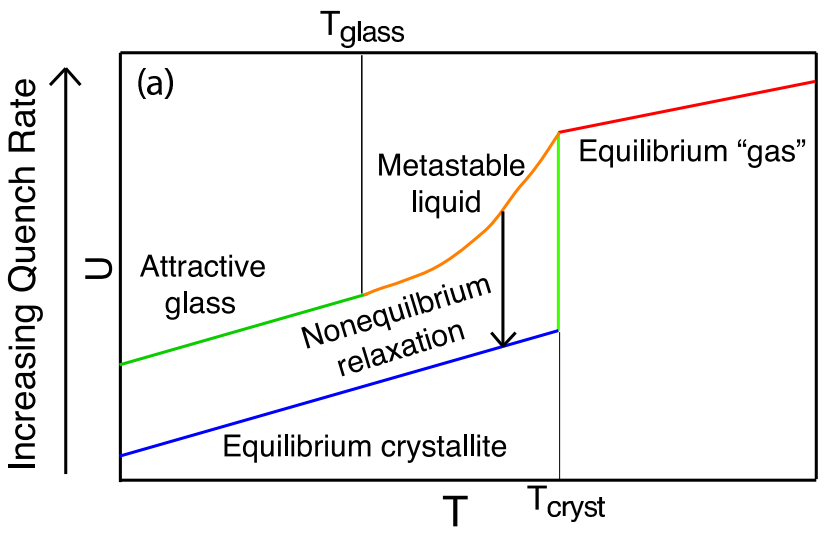

Fig. 1 Schematic of potential energy versus temperature $T$ for systems with short-range attractive interactions cooled at varying quench rates $\dot{T} .11$ We compare the nonequilibrium and near-equilibrium crystallization dynamics of polymers and colloids by quenching systems at various rates and observing relaxation dynamics below $T_{\text {cryst }}$.

to chain topology. Near-equilibrium crystallites possess similar static structure when $T$ is scaled by the equilibrium crystallization temperature $T_{\text {cryst }}$. Relaxation dynamics at fixed $T / T_{\text {cryst }}$ are qualitatively similar, but quantitatively quite different for polymers and colloids. Using multiple measures of crystalline order and particle rearrangements, we quantify how restrictions on local motion imposed by the covalent backbone slow the approach to equilibrium by eliminating 'monomeric' relaxation mechanisms. Rearrangements in polymeric crystallites are required to be more cooperative, and hence are slower, than in their colloidal counterparts. We validate these results by examining whether these trends vary significantly with system size (i.e. chain length) $N$. For $N$ varying over a range typically studied in single chain polymer crystallization experiments,, 2 increasing system size does not affect qualitative trends, but quantitatively strengthens them.

The outline of the rest of the paper is as follows. In Section 2. we describe our model, simulation protocol, and metrics used to analyze our data. Section 3 presents results for thermal quenches over a wide range of $|\dot{T}|$, nonequilibrium evolution of crystallite properties at fixed $T_{f}<T_{\text {cryst }}$, and a detailed comparison of rearrangements in polymers and colloids. Finally, in Section 4, we summarize our findings and place them in context of other recent experimental and simulation studies.

\section{Methods}

Recent Monte Carlo simulations have employed advanced sampling techniques, such as topology-changing bridging moves, to investigate the equilibrium phase behavior 1.2 of single-chain polymers. In contrast, molecular dynamics (MD) simulations with physically realistic dynamics are better able to capture the complex, coordinated rearrangement events associated with the glassy dynamics of crystallization.

In our studies, both colloidal and polymeric systems consist of $N$ identical spherical monomers that interact via the harmonic "sticky-sphere" potential shown in Fig.2(a):

$$
U_{\text {harm }}(r)=\left\{\begin{array}{cc}
-\epsilon+\frac{k}{2}\left(\frac{r}{D}-1\right)^{2}, & r<r_{c} \\
0 & , r>r_{c}
\end{array},\right.
$$

where $\epsilon$ is the intermonomer binding energy, $D$ is the monomer diameter, and $k=1600 \epsilon$ is the spring constant. The only difference between colloidal and polymeric interactions (inset to Fig.2[a)) is that in polymeric systems, the monomers are linked into a linear chain connected by $N-1$ permanent covalent bonds. $\frac{13}{=}$ Different values for $r_{c}$ are used for covalently and noncovalently bonded monomers: $r_{c}^{c} / D=\infty$ and $r_{c}^{n c} / D=1+\sqrt{2 \epsilon / k}$, respectively.

Newton's equations of motion are integrated using the velocity-Verlet method with a timestep $d t=\tau / 800$, where $m$ is the monomer mass and $\tau=\sqrt{m D^{2} / \epsilon}$. We determined that this timestep was sufficiently small by examining $d t$-dependence of the velocity autocorrelation function $v_{a c}(t)$ in simulations at high temperature; no statistically significant dependence was found for $d t \leq \tau / 600$. Below, we express length scales, energies, times, rates and temperatures in units of $D, \epsilon, \tau, \tau^{-1}$, and $\epsilon / k_{B}$, respectively. The temperature $T$ is controlled via a Langevin thermostat with damping time 10. A periodic cubic simulation cell with volume $L^{3}$ fixes the monomer density $\rho$. We present results for $\rho=0.01$, which is in the dilute limit.

Systems are initialized in random walk initial conflgurations (for polymers) and random nonoverlapping initial positions (for colloidal systems). They are then equilibrated at high temperature, $k_{B} T_{i} / \epsilon=0.75$, for times long compared to the time over which the self intermediate scattering function decays to zero. At $T=T_{i}$, polymeric systems are in the good-solvent (self-avoiding coil) limit, and colloidal systems are in an ideal-gas-like state. Following equilibration, systems are thermally quenched at various rates $\dot{T}$. In our ensembleaveraging approach, states from which thermal quenches are initiated are separated by times long compared to structural relaxation times, and thus are statistically independent.

The quenches are either continued to $T=0$ or terminated at $T=T_{f}<T_{\text {cryst. }}$. In the latter case, we continue the runs at fixed $T_{f}$. We choose $T_{f} / T_{\text {cryst }}=7 / 8$ to suppress finite- $N$ fluctuation effects associated with thermal broadening of the phase transition to crystallites, $\underline{\underline{15}}$ i.e. $T_{f} / T_{\text {cryst }} \lesssim 1-N^{-1 / 2}$, yet allow sufficiently fast relaxation to be captured within the limits of available computational resources for the $N$ considered here $(40,100$, and 250). As we 
will show below, this procedure yields particularly interesting results for nonequilibrium relaxation following quenches at moderate $|\dot{T}| . T_{f} / T_{\text {cryst }}=7 / 8$ is also comparable to temperatures used in many experimental and simulation studies of crystallite nucleation and growth in supercooled colloidal and polymeric systems. 16,17

We will examine several order parameters to characterize nucleation, growth and rearrangements of crystallites as a function of temperature and time following thermal quenches to $T_{f} / T_{\text {cryst }}<1$. These order parameters are generated from the adjacency matrix $\bar{A} ; A_{i j}=A_{j i}=1$ when particles $i$ and $j$ are in contact, i.e. when the position vectors satisfy $\left|\vec{r}_{i}-\vec{r}_{j}\right|<r_{c}^{n c}$, and 0 otherwise.

Our interaction potential (Eq. 11) promotes contactdominated crystallization dynamics. $\frac{18}{}$ For the large $k / \epsilon$ and small $r_{c}$ employed here, the ground states for colloidal and polymeric systems 19 are simply the states that maximize the number of pair contacts $N_{c}=\Sigma_{j>i} A_{i j}$ for a given $N . \underline{20-22}$ Further, our systems form crystallites possessing close-packed cores that increase in size as $T$ decreases or equilibrium is approached. $\underline{\underline{18}}$ We therefore measure the number of close packed monomers $N_{c p}=\Sigma_{i=1}^{N} \delta\left(\Sigma_{j} A_{i j}-12\right)$ and degree distribution $P(\eta)$, i.e. the probability for a particle to have $\eta$ contacts. Note that $\sum_{i=0}^{12} i P(i)=2 N_{c} / N$ and $P(12)=N_{c p} / N . P(\eta)$ contains more information than $N_{c}$ and $N_{c p}$ since it describes the high- $\eta$ cores of crystallites as well as their surfaces, where monomers naturally have lower $\eta$ * We will argue below that the combination of $N_{c}, N_{c p}$ and $P(\eta)$ forms an effective set of "crystal-agnostic" order parameters (in the spirit of Rein ten Wolde et.al. .16 ).

We will also present results for the adjacency matrix autocorrelation function $P_{\mathrm{AMAC}}\left(t_{w}, t^{\prime}\right)$ as a function of $t^{\prime}$ after waiting various times $t_{w}$ following thermal quenches to $T_{f}$ :

$$
P_{\mathrm{AMAC}}\left(t_{w}, t^{\prime}\right)=\left\langle\frac{\sum_{i, j>i} A_{i j}\left(t_{w}\right) A_{i j}\left(t_{w}+t^{\prime}\right)}{\sum_{i, j>i} A_{i j}\left(t_{w}\right) A_{i j}\left(t_{w}\right)}\right\rangle,
$$

where the brackets indicate an ensemble average over independently prepared samples, and the total time elapsed after termination of the quench is $t=t_{w}+t^{\prime}$. For polymers, we exclude the contributions of covalent bonds to $P_{\mathrm{AMAC}}$ by summing over $j>i+1$ rather than $j>i$ in Eq. 2. Both $P_{\text {AMAC }}\left(t^{\prime}\right)$ and the intermediate scattering function $S\left(q, t^{\prime}\right)$ evaluated at $q D \simeq 2 \pi$ identify rearrangements of contacting neighbors, which control the slow relaxation processes in colloidal systems with competing crystallization and glass transitions. ${ }^{27,28}$ To capture the glassy dynamics, we examine systems with $t_{w}$ ranging over several orders of magnitude and $t^{\prime} \gg t_{w}$.

* $P(\eta)$ is also closely related to the spectrum of eigenvalues of $\bar{A}$. 26 While these eigenspectra provide additional information on crystallite structure, we leave their examination for future studies of equilibrium systems.

\section{Results}

In this section, we compare the collapse and ordering dynamics of colloids and polymers using two protocols. Protocol (1) consists of decreasing the temperature from an initially high value $T_{i}$ to zero using a wide range of thermal quench rates $\dot{T}$. To analyze changes in structure with decreasing $T$, we measure the potential energy, $N_{c}$, and $N_{c p}$ over the fullrange of temperature for each quench rate. Protocol (2) consists of quenching systems from $T_{i}$ to $T_{f}=(7 / 8) T_{\text {cryst }}$ using a range of $\dot{T}$ and then monitoring structural order and rearrangement events within crystallites at $T=T_{f}$ as systems evolve toward equilibrium. We measure $N_{c}(t), N_{c p}(t)$, $P(\eta ; t)$ and $P_{A M A C}\left(t_{w}, t^{\prime}\right)$, to quantify evolution to more ordered states, and also perform detailed studies of crystallite rearrangements in a "pre-terminal" relaxation regime where the systems slowly approach equilibrium.

\subsection{Protocol 1: Thermally Quench from High to Zero $T$}

Potential Energy: Figure 2 shows results for the scaled potential energy $U / N \epsilon$ for colloidal and polymer systems quenched from $T=T_{i}$ to zero. Panel (a) shows results at low $|\dot{T}|$ for system sizes ranging over a factor of six in $N$, while panel (b) shows results for $N=100$ over a range of $|\dot{T}|$ spanning four orders of magnitude. All results are consistent with the general picture of Fig. 1, and illustrate both features common to polymers and colloids as well as differences arising from the presence of a covalent backbone.

At the lowest $|\dot{T}|$ considered $\left(\sim 10^{-8}\right)$, both colloids and polymers show sharp, first-order-like 1 transitions at corresponding $T=T_{\text {cryst }}$. Because of the narrowness of the attractive range of the potential well,,$\frac{1,29}{.}$ no intermediate liquid state (i.e., globules in the case of polymers) appears $\frac{18,30}{}$. In both cases, as in Fig. 1, the equilibrium transitions are from gas-like states to crystallites. No significant quench rate dependence is observable for $T>T_{\text {cryst }}$, which indicates that all $|\dot{T}|$ are sufficiently low to be near-equilibrium in this high temperature regime. For $|\dot{T}| \sim 10^{-8}$, polymers and colloids have the same energy at low $T$ to within statistical noise, showing that this quench rate is slow enough to be in the near-equilibrium limit for polymers (i.e. $\left|\dot{T}^{*}\right| \gtrsim 10^{-8}$ for these systems.) The $N$-dependence (panel (a)) shows only quantitative rather than qualitative differences. As $N$ increases, values of $U / N \epsilon$ in the $T \rightarrow 0$ limit decrease because larger crystallites possess more interior monomers.

Compared to colloids, polymers have lower $U / N \epsilon$ for $T>$ $T_{\text {cryst }}$ because of the permanent covalent bonds, which contribute $\sim k_{B} T / \epsilon-1 . \stackrel{30}{3}$ They also have higher absolute values of $T_{\text {cryst }}$ (Table 1 ). To zeroth order, $T_{\text {cryst }}^{\text {pol }} / T_{\text {cryst }}^{\text {coll }} \simeq 3 / 2$, a ratio which can be explained by a simple degree-of-freedom counting argument: while colloids have $3 N-6$ nontrivial 

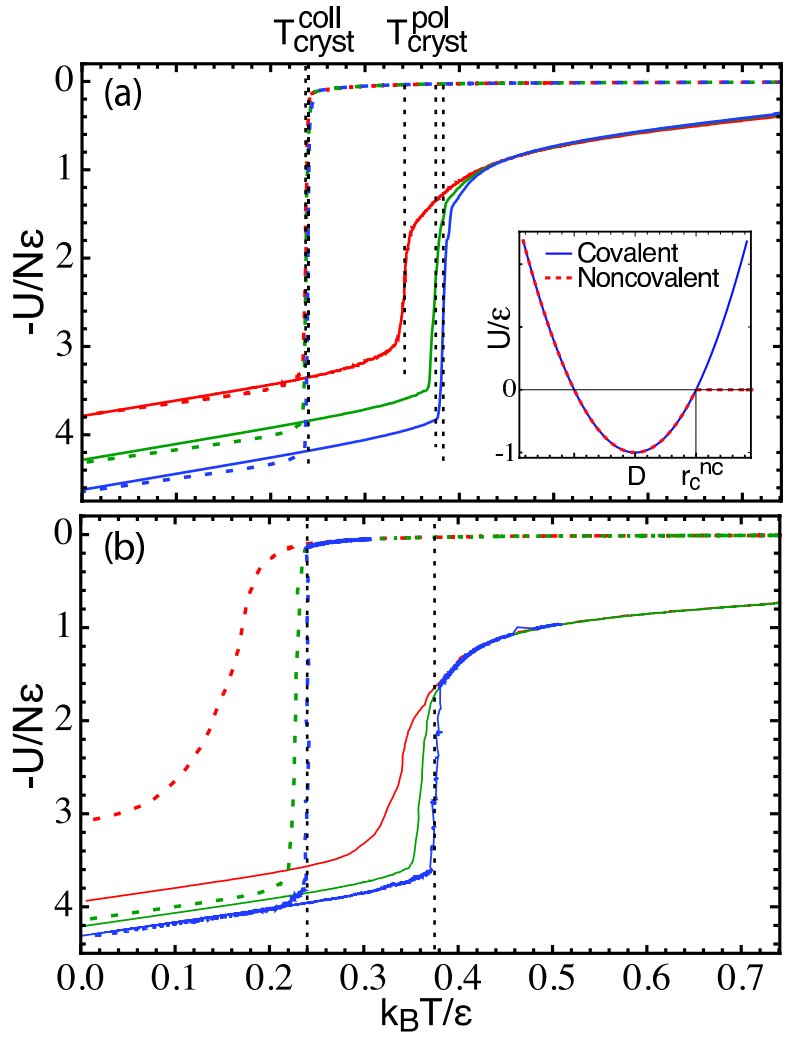

Fig. 2 (a) System size dependence and (b) quench rate dependence of potential energy versus temperature from molecular dynamics simulations of polymers (solid curves) and colloids (dashed curves). Vertical dashed lines indicate $T_{\text {cryst. }}$. Panel (a) shows results for a single slow $\left(|\dot{T}|=2.5 \cdot 10^{-8}\right)$ quench rate for $N=40$ (red), $N=100$ (green), and $N=250$ (blue). Panel (b) shows results for $N=100$ systems. Colors indicate $|\dot{T}|=10^{-4}$ (red), $10^{-6}$ (green) and $10^{-8}$ (blue). Results in (a) are averaged over 40 statistically independent samples, while results in (b) are averaged over 104 statistically independent samples for the higher two quench rates and 8 for the lowest. The inset to (a) illustrates the interaction potential (Eq. 1) employed in our simulations. degrees of freedom, the stiff covalent bonds in polymers act like holonomic constraints, reducing the effective dimensionality of their phase space to $2 N-5$ so that $T_{\text {cryst }}^{\text {pol }}=[(3 N-$ $6) /(2 N-5)] T_{\text {cryst }}^{\text {coll }} \simeq 3 / 2 .{ }^{31}$ This observation helps motivate our (protocol 2) studies comparing relaxation of polymeric and colloidal crystallites at equal values of $T / T_{\text {cryst }}$ in terms of their different free energy landscapes.

Table 1 Dependence of $T_{\text {cryst }}$ on $N$ and topology. Our data are consistent with detailed analyses ${ }^{\frac{8}{-}}$ predicting $O\left(N^{-1 / 3}\right)$ finite-size corrections to $T_{\text {cryst }}^{\text {pol }}$.

\begin{tabular}{llcc}
\hline$N$ & $T_{\text {cryst }}^{\text {coll }}$ & $T_{\text {cryst }}^{\text {pol }}$ & $T_{\text {cryst }}^{\text {pol }} / T_{\text {cryst }}^{\text {coll }}$ \\
\hline 40 & 0.237 & 0.342 & 1.44 \\
100 & 0.240 & 0.375 & 1.56 \\
250 & 0.239 & 0.383 & 1.60 \\
\hline
\end{tabular}

Below $T_{\text {cryst }}$, dramatic quench rate dependence appears. Consistent with the general picture of Fig. 1, with increasing $|\dot{T}|$, the $T$-dependent potential energy increases relative to the equilibrium-crystal limit. Figure 2 (b) shows results for $N=100$. Similar trends are observed for the other $N$ considered, with the main difference being that $\left|\dot{T}^{*}\right|$ increases and rate effects for $|\dot{T}|>\left|\dot{T}^{*}\right|$ become more dramatic with increasing $N$.

Contact number: We now discuss the temperature dependence of local measures of structure during quenches. Figure 3 shows $N_{c}(T)$ and $N_{c p}(T)$ plotted against $T$ and $T / T_{\text {cryst }}$ for the same systems analyzed in Fig. 2(b). $N_{c} / N$ and $N_{c p} / N$ are particularly sensitive measures of crystallite equilibration. They increase monotonically with decreasing $|\dot{T}|$, but the variations with rate are different for polymers and colloids. For fast quenches, polymers are more efficient crystal formers due to topological connectivity and the resultant cooperative dynamics. ${ }^{7}$ An example of a cooperative polymer collapse mechanism not found in colloids is "chain pull-in"; a nucleus forms between chemically nearby monomers, and covalent bonds pull in segments that are chemically adjacent to the nucleus. ${ }^{33}$ However, as exemplified by the $|\dot{T}|=10^{-4}$ results, this mechanism produces only small ordered cores with highly disordered exteriors. Note that for the fastest quench rates, the collapsed structures possess $N_{c} / N \geq 3$, which is the minimal number of contacts for mechanical stability ${ }^{32}$ and corresponds to the isostaticity threshold shown in panel (a).

In Fig. 3(a), we show that $\left|\partial N_{c} / \partial T\right| \rightarrow 0$ at a noticeably higher $T$ for polymers than colloids, which indicates that large structural rearrangements cease, and polymers possess a higher $T_{\text {glass }}$ than colloids. ${ }^{27}$ Polymer chain backbones follow tortuous paths through the interior of collapsed states 21,40 and chain uncrossability suppresses large rearrangements, so a higher $T_{\text {glass }}$ is consistent with the expected slower relaxation dynamics for polymer crystallites. 

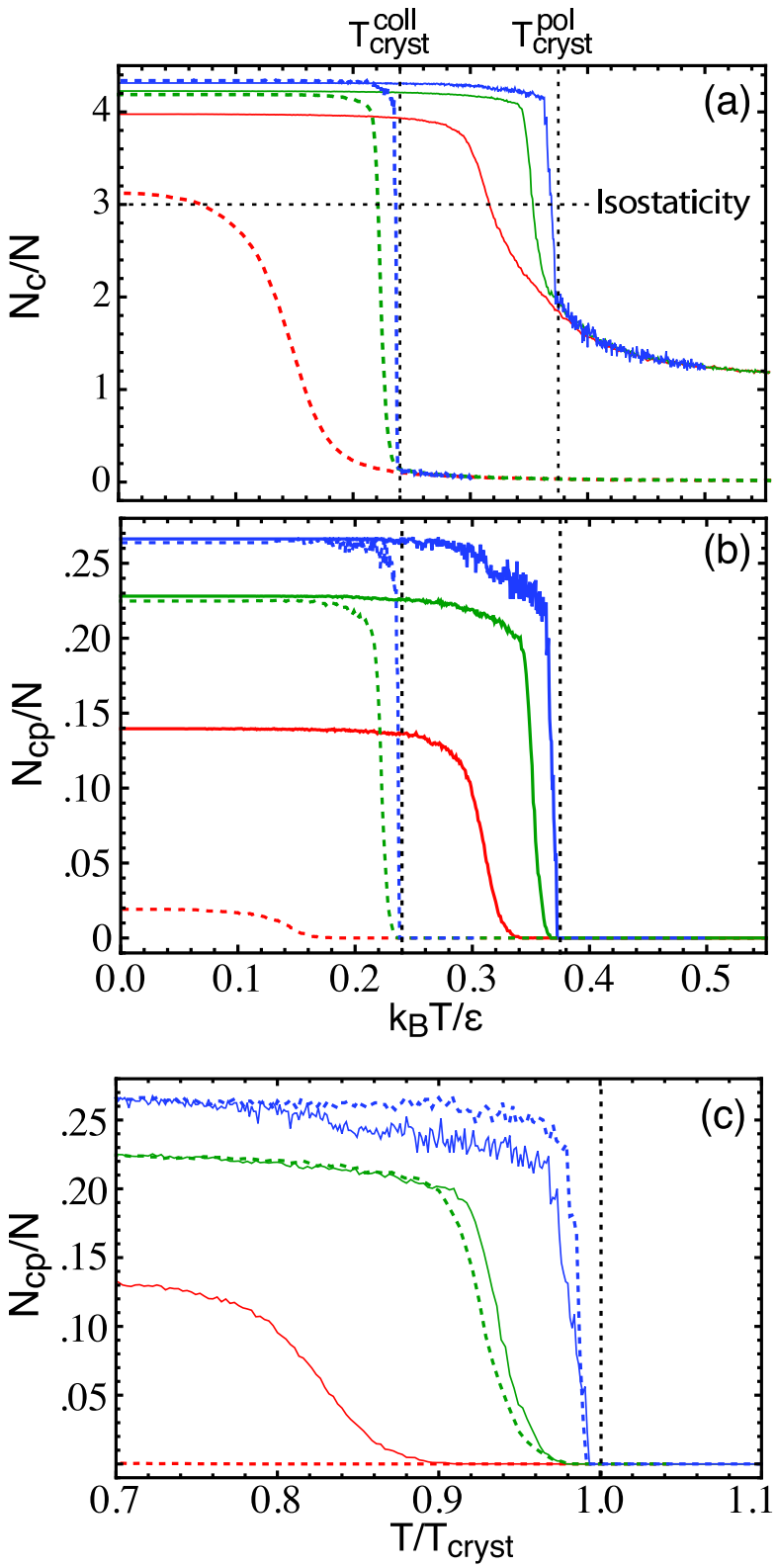

Fig. 3 Measures of local structural order, (a) $N_{c} / N$ and (b) $N_{c p} / N$, as a function of temperature during thermal quenches from $T_{i}=0.75$ to $T_{f}=0$. (c) Number of close-packed spheres $N_{c p} / N$ plotted versus temperature reduced by the crystallization temperature, $T / T_{\text {cryst }}$. The line color, dashing scheme, and $(N=100)$ systems studied are the same as those in Fig. 2 b). Vertical dashed lines indicate $T_{\text {cryst }}$ for colloids and polymers, and the horizontal dashed line in panel (a) indicates the isostaticity ${ }^{32}$ threshold $\left(N_{c} / N=3\right)$. We define $T_{\text {glass }}$ as the temperature at which rearrangements cease during a constant rate quench and the slope $\partial N_{c} / \partial T$ becomes close to zero.
We find that measures of energy and contact number ( $U, N_{c}$, and $N_{c p}$ ) plotted against the scaled temperature $T / T_{\text {cryst }}$ for polymers and colloids all increasingly collapse as $|\dot{T}|$ decreases. Figure 3(c) shows $N_{c p}\left(T / T_{\text {cryst }}\right)$ for different quench rates. Over a broad range of $T$, the collapse of ensemble-averaged values of $N_{c}\left(T / T_{\text {cryst }}\right)$ and $N_{c p}\left(T / T_{\text {cryst }}\right)$ for $N=40$ and $N=250$ is of similar quality $\dagger^{\dagger}$ This is a nontrivial result for the small crystallites considered here, where ordering and surface-to-volume ratio changes with $N$ and finite-size effects are in principle important. As shown in Table 2, terminal values of $\left\langle N_{c} / N\right\rangle$ increase from $\sim 3.8$ to $\sim 4.7$ as $N$ increases from 40 to 250 and values of $\left\langle N_{c p} / N\right\rangle$ at $T=0$ increase even more (from $\sim 0.15$ to $\sim 0.35$ ) as the volume-to-surface-area ratio of crystallites increases and they form larger close-packed cores. The collapse of $U\left(T / T_{\text {cryst }}\right), N_{c}\left(T / T_{\text {cryst }}\right)$, and $N_{c p}\left(T / T_{\text {cryst }}\right)$ despite these $N$-dependent changes in order suggests that polymer and colloid crystallites, in equilibrium, occupy similar positions on their respective energy landscapes at equal values of $T / T_{\text {cryst }}$ even though the absolute $T$ are different.

Table 2 Variation with $N$ of $\left\langle N_{c}\right\rangle$ and $\left\langle N_{c p}\right\rangle$ during $|\dot{T}|=2.5 \cdot 10^{-8}$ quenches. Results are from the same systems depicted in Fig. 2 2 a), and values of $T_{\text {cryst }}$ are given in Table 1 Results for $\left\langle N_{c}\right\rangle / N$ are consistent with leading order $O\left(N^{-1 / 3}\right)$ corrections away from the $N \rightarrow \infty$ value (6).

\begin{tabular}{lccccc}
\hline$N$ & $T$ & $\left\langle N_{c}^{\text {coll }} / N\right\rangle$ & $\left\langle N_{c}^{\text {pol }} / N\right\rangle$ & $\left\langle N_{c p}^{\text {coll }} / N\right\rangle$ & $\left\langle N_{c p}^{\text {pol }} / N\right\rangle$ \\
\hline 40 & $\frac{7}{8} T_{\text {cryst }}$ & 3.73 & 3.68 & 0.143 & 0.130 \\
40 & 0 & 3.78 & 3.76 & 0.150 & 0.146 \\
\hline 100 & $\frac{7}{8} T_{\text {cryst }}$ & 4.31 & 4.24 & 0.259 & 0.239 \\
100 & 0 & 4.33 & 4.29 & 0.265 & 0.255 \\
\hline 250 & $\frac{7}{8} T_{\text {cryst }}$ & 4.67 & 4.60 & 0.362 & 0.329 \\
250 & 0 & 4.69 & 4.65 & 0.368 & 0.343 \\
\hline
\end{tabular}

\subsection{Protocol 2: Thermally quench from high $T$ to $T_{f}<$ $T_{\text {cryst }}$ and monitor relaxation at fixed $T_{f}$}

As shown in Table 2, polymeric and colloidal crystallites of the same $N$ have nearly equal values of both $\left\langle N_{c} / N\right\rangle$ and $\left\langle N_{c p} / N\right\rangle$ at $T_{f}=(7 / 8) T_{\text {cryst }}$. They also have similar structure (i.e. $P(\eta)$; $c f$. Fig. 5). This helps establish that comparison of relaxation dynamics in polymeric and colloidal crystallites at fixed $T=T_{f}$ is a reasonable approach to isolating the

$\dagger$ Collapse worsens only slightly with increasing $N$, indicating that $\left|\dot{T}^{*}\right|$ increases slowly with $N$. Precise calculation of $\left|\dot{T}^{*}(N)\right|$ is outside the scope of this study, but more quantitative results for the $N$-dependence of characteristic relaxation times is given in Section 3.2

$\ddagger$ This variation is significant when one considers that the isostatic value $\left\langle N_{c} / N\right\rangle=3$ can be attained by systems as small as $N=16,34$ while the limiting value for infinite $N$ (corresponding to defect-free close-packed crystals) is $\left\langle N_{c} / N\right\rangle=6$. 
role played by topology in controlling the approach of crystallites to equilibrium.

We now examine systems quenched at different rates and monitor their evolution as a function of time $t$ following termination of the quenches to $T_{f}$. Measures such as the evolution of contact order and several measures of local and nonlocal rearrangements after different waiting times $t_{w}$ show that polymeric crystallites possess significantly slower long-time relaxation dynamics at equal values of $T / T_{\text {cryst }}$ despite their similar structure. Studies of system size dependence show that this "topological" slowdown is related to the more correlated rearrangements imposed by uncrossable covalent backbones, and strengthens with increasing $N$.

Contact number: Fig. 4 shows the evolution of $\delta N_{c},\left\langle N_{c}\right\rangle$, and $\left\langle N_{c p}\right\rangle$ in polymeric and colloidal systems as a function of time $t$ following thermal quenches to $T_{f}$ at rates $|\dot{T}|=$ $10^{-6}$ and $10^{-7} . \delta N_{c}$ is the root-mean-square fluctuation in the number of contacts averaged over an ensemble of collapse trajectories. The strong increase in $\left\langle N_{c}\right\rangle$ and $\left\langle N_{c p}\right\rangle$ and drop in $\delta N_{c}$ after $t \simeq 10^{5}$ for $|\dot{T}|=10^{-6}$ in Fig. 4(a) suggests that the crystal nucleation rate for colloids is $r_{c} \approx 10^{-5}$. In contrast, polymers do not show a rapid increase in the number of contacts (or concomitant decrease in the contact number fluctuations) at these thermal quench rates, showing that the crystal nucleation rate for $(N=100)$ polymers is $r_{p} \gtrsim 10^{-5}$.

As shown in Fig. 4 (b) and (c), at long times $t>10^{6}$ both polymeric and colloidal systems show evidence of logarithmic relaxation toward equilibrium. The logarithmic increase in $\left\langle N_{c}(t)\right\rangle$ and $\left\langle N_{c p}(t)\right\rangle$ is consistent with thermal activation over large energy barriers and transitions between metastable, globule-like states and near-equilibrium crystallites. $\stackrel{1,4}{ }$ Relaxation is also slowed by the increasing mechanical rigidity associated with increasing $N_{c}$. 35 The approach to equilibrium occurs through thermally-activated rearrangements of particles associated with "soft modes" ( $c f$. Figs. 7, 8), which are known to play a significant role in structural and stress relaxation in supercooled liquids. 36 At larger $t$, the slopes (indicated by green solid lines) are clearly larger for colloids than for polymers; values of $\partial\left\langle N_{c}\right\rangle / \partial \ln _{10} t$ and $\partial\left\langle N_{c p}\right\rangle / \partial \ln _{10} t$ fit over the range $10^{6.5} \leq t \leq 10^{7}$ are given in Table 3 .

For $t>10^{7}$, values of $\partial\left\langle N_{c}(t)\right\rangle / \partial \log _{10}(t)$ and $\partial\left\langle N_{c p}(t)\right\rangle / \partial \log _{10}(t)$ decrease for colloidal systems as they enter a terminal relaxation regime associated with ergodic exploration of their full free energy landscape. The approach to the ergodic limit can be clearly seen in the vanishing of "history" dependence for systems quenched at different rates, i.e. curves for different $\dot{T}$ overlap at large $t$. In contrast, for polymers, history dependence and faster logarithmic relaxation persist up to the maximum time $t=5 \cdot 10^{7}$. This shows that polymers possess slower characteristic relaxation rates $s_{\text {slow }}$ at the same value of $T / T_{\text {cryst }}$ even though the crystallites are less mechanically rigid (since they have fewer contacts and are
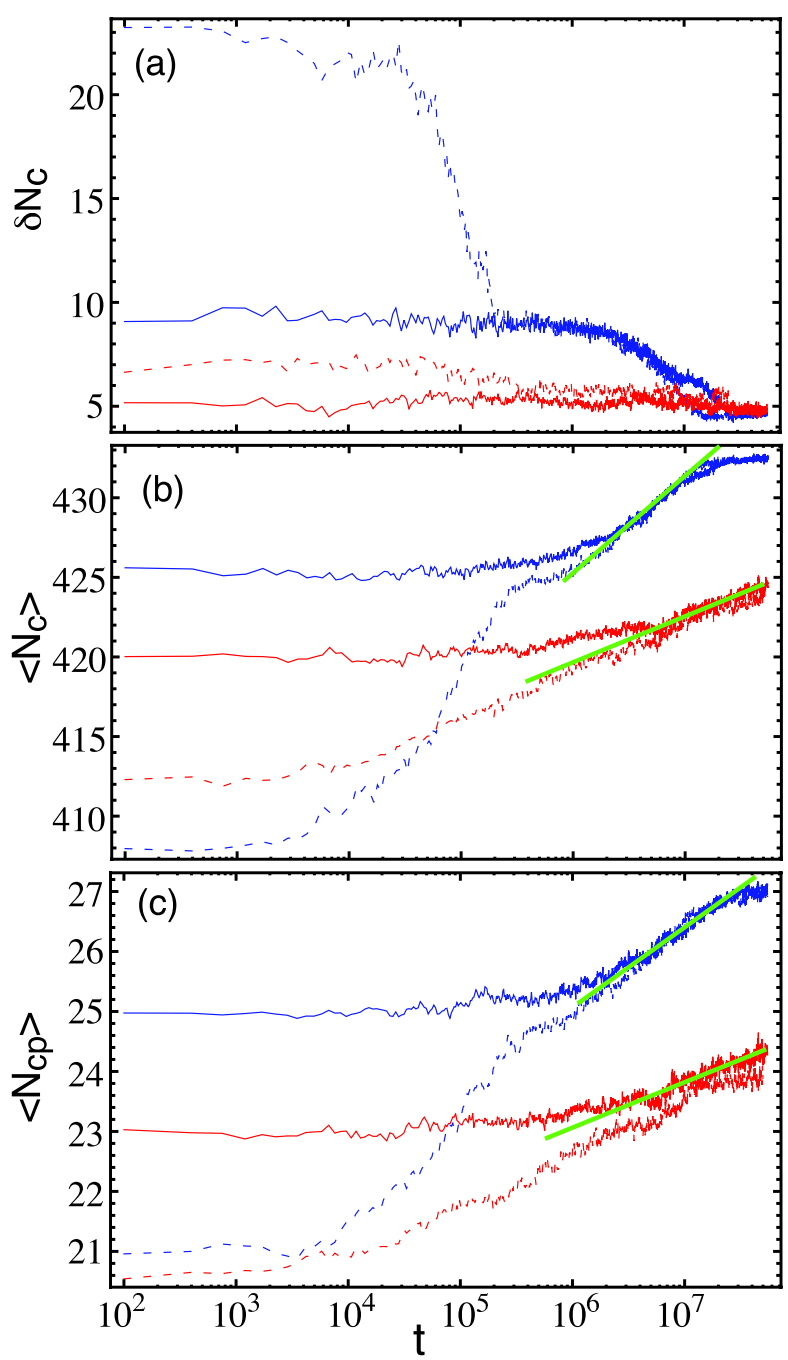

Fig. 4 Nonequilibrium structural relaxation for polymeric (red) and colloidal (blue) systems after thermal quenches from $T_{i}>T_{\text {cryst }}$ to $T_{f}=(7 / 8) T_{\text {cryst }}$ at $|\dot{T}|=10^{-6}$ (heavier solid lines) and $10^{-7}$ (lighter dashed lines). Results are averaged over 104 independent $N=100$ samples and are plotted as a function of time $t$ following the termination of the quenches: (a) Standard deviation in the number of contacts $\delta N_{c}=\left\langle N_{c}^{2}\right\rangle-\left\langle N_{c}\right\rangle^{2}$, (b) mean number of contacts $\left\langle N_{c}\right\rangle$, and (c) mean size of the close-packed cores $\left\langle N_{c p}\right\rangle$. In (b) and (c), the solid green lines show fits to logarithmic behavior at long times ( $c f$. Table 3); the lines are extended as a guide to the eye. 
at higher absolute $T$ ).

While observation of the crossover into this terminal relaxation regime for polymers with $N=100$ and $N=250$ monomers is made unfeasible by the limitations of current computer power, in this paper we are primarily concerned with the nonequilibrium dynamics of crystallization and the logarithmic "pre-terminal" relaxation regime of crystallite growth. We now analyze the role of topology on relaxation dynamics within the pre-terminal regime by examining the evolution of more detailed measures of crystalline order.

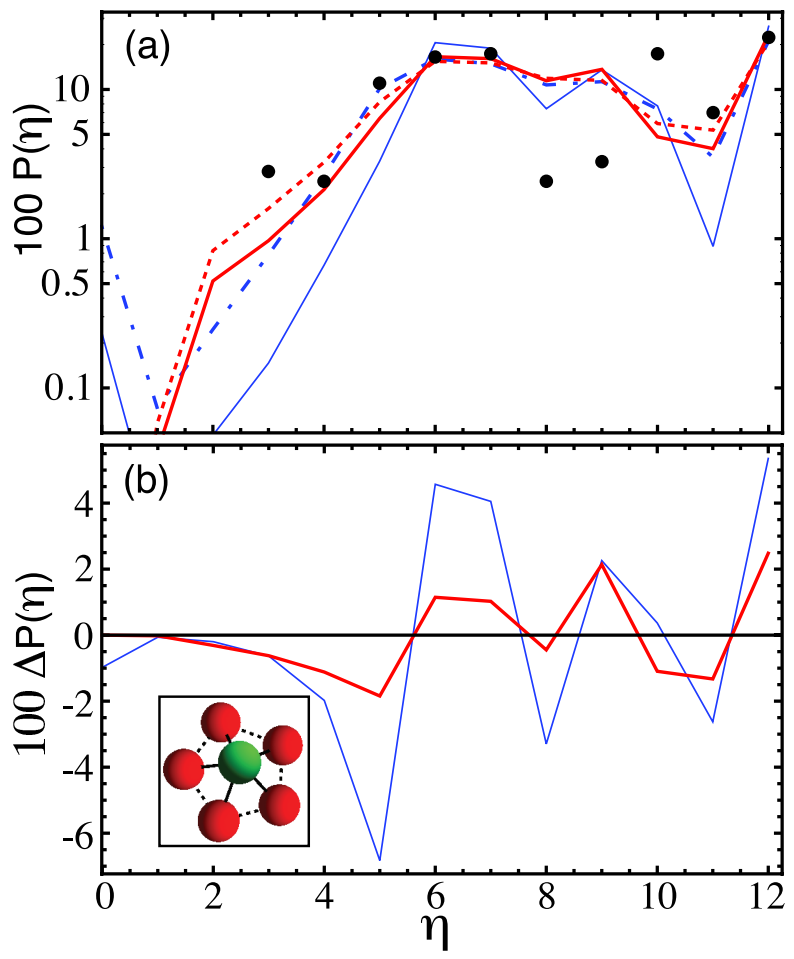

Fig. 5 Evolution of degree distributions $P(\eta)$. (a) $P(\eta)$ after $|\dot{T}|=10^{-6}$ quenches to $T_{f}$. Curves show data averaged over times $0 \leq t \leq 10^{4}$ for colloids (dash-dotted) and polymers (dashed) and $0.999 \cdot 10^{7} \leq t \leq 10^{7}$ (colloids; light solid, polymers; heavy solid). Solid circles show results averaged over the 6 distinct $N=100$ Barlow packings. ${ }^{37}$ Panel (b) shows $\Delta P(\eta)$, obtained by subtracting the small- $t$ data shown in panel (a) from large- $t$ data for colloids (lighter blue line) and polymers (heavier red line). Inset: A common five-fold symmetric structure present on the surface of nonequilibrium crystallites. The green monomer has $\eta=5$.

Degree distribution: Figure 5 shows results for the evolution of $P(\eta)$ following $|\dot{T}|=10^{-6}$ quenches. Polymeric and colloidal crystallites have similar degree distributions for intermediate and high $\eta$, indicating the crystallites' inner cores are similarly structured. However, covalent backbones produce greater differences at crystallite surfaces. Polymer topology requires $\eta \geq 2$ for chemically interior monomers and $\eta \geq 1$ for chain ends, while monomers in colloidal systems can have any degree of connectivity consistent with steric constraints (here, $0 \leq \eta \leq 12$ ). Because of this difference in connectivity, polymer crystallites include a higher fraction of monomers with $2 \leq \eta \leq 4$ (panel (a)); this difference strengthens as $t$ increases and systems approach equilibrium.

Panel (b) shows $\Delta P(\eta)=P\left(\eta ; t=10^{7}\right)-P(\eta ; t=0)$ to highlight the changes in $P(\eta ; t)$ over an interval $\Delta t=10^{7}$ following the end of the quench to $T_{f}$. Notable features common to colloidal and polymeric crystallites and depicted more clearly than in panel (a) include sharply negative $\Delta P(5)$ associated with the annealing out of 5-fold symmetric structures that tend to form on the surface of metastable crystallites (see inset), and sharply negative $\Delta P(11)$ associated with annealing out of defects within crystallite cores. The latter process is closely associated with the increase in $\left\langle N_{c p}(t)\right\rangle$ shown in Fig. 4. However, the key result shown here is that changes in $P(\eta)$ are uniformly smaller for polymers despite the higher absolute $T$.

While the equilibrium $P(\eta)$ distribution is difficult to calculate for large $N$ without resorting to advanced Monte Carlo techniques, $, 1,2$ one measure of evolving crystalline order in our systems can be obtained by comparing $P(\eta ; t)$ to that of a known reference system. Barlow packings 38 are hardsphere packings composed of layered hexagonal-close-packed planes; their three-dimensional order may be fcc, hcp, or mixed fcc/hcp, but they possess no defects (e.g. stack faults.) These are "reference" low energy structures for our model in the limit of steep hard-core repulsions and short-range attractions. The solid circles in Fig. 5(a) show $P(\eta)$ averaged over the six $N=100$ Barlow packings. 37

Our simulation data show that crystallites at the end of the pre-terminal relaxation regime possess Barlow-like order. Interestingly, $\left\langle N_{c}\right\rangle$ is slightly higher (Fig. 4) in the simulated systems than in the Barlow packings $\left(\left\langle N_{c}^{\text {Barlow }}\right\rangle=421\right)$, while values of $P(12)=\left\langle N_{c p} / N\right\rangle$ are similar. These effects are attributable to finite temperature, stiffness of core repulsions and range of attractive interactions. Other differences between our systems and Barlow packings are attributable to small deviations from equilibrium, the fact that our crystallites may be stack-faulted, and (for polymers) entropic factors such as blocking. 21 That the above complexity can be understood by examining $N_{c}, N_{c p}$, and $P(\eta)$ shows that this combination of metrics constitutes an effective "crystal-agnostic" 16 measure of order $\$$

In the above protocol 2 subsections, we have focused on results for $N=100$. System size effects are minor, e.g. slower logarithmic growth of crystalline order with increasing $N$ and shift of $P(\eta)$ towards higher $\eta$. Examining rear-

$\S$ Indeed, previous coarse-grained Monte Carlo studies of polymer crystallization ${ }^{39-41}$ focusing on dense bulk systems have employed similar structural measures. 
rangements within crystallites provides additional insight into $N$ - and topology-dependent effects on the glassy dynamics of crystallite formation and is discussed in the following subsections.

Adjacency matrix autocorrelation function: Next we examine the decorrelation of contacts between neighboring particles during the approach to equilibrium at $T_{f}=(7 / 8) T_{\text {cryst }}$. We first examine effects of quench rate and waiting time on $N=100$ systems, and then examine $N$-dependence for evolution following slow quenches. The adjacency matrix autocorrelation function $P_{\mathrm{AMAC}}\left(t_{w}, t^{\prime}\right)$ displays several important features (Fig. (6):

(i): Polymeric rearrangement events are more frequent at low $t^{\prime}$ because of the higher absolute $T$. However, rearrangements are slower at large $t^{\prime}$ despite the higher absolute $T$. The slower decay arises from the covalent bonds in polymers that restrict the motion of monomer $i$ to the plane tangent to the vector $\vec{r}_{i+1}-\vec{r}_{i-1}$. Although contributions from permanent covalent bond contacts are excluded from the definition of $P_{\text {AMAC }}\left(t_{w}, t^{\prime}\right)$, in compact crystallites connectivity to chemically distant monomers produces long-range suppression of contact-breaking

(ii): Following the $|\dot{T}|=10^{-6}$ thermal quenches, $P_{\text {AMAC }}\left(t_{w}, t^{\prime}\right)$ for both polymers and colloids display strong $t_{w}$-dependence as shown in Fig. 6(a). We find an increase in the length and height of the low- $t^{\prime}$ "plateau" near $P_{\text {AMAC }}\left(t_{w}, t^{\prime}\right) \approx 0.95$ with increasing $t_{w}$, similar to the behavior of the plateau in $S\left(q, t_{w}, t^{\prime}\right)$ during the aging process in structural glasses. ${ }^{27}$ Aging effects are stronger for colloids than for polymers because colloids are further from equilibrium at the termination of the quenches (Fig. 4(a)).

(iii): For slower thermal quenches $\left(|\dot{T}|=10^{-7}\right.$; Fig. 6(b)), similar but much weaker aging effects are present. Results for $t_{w}=0$ and $t_{w}=10^{5.5}$ are indistinguishable to within statistical uncertainties for both polymers and colloids. Aging is delayed in part because the additional time to quench from $T_{\text {cryst }}$ to $T_{f}$ provided by the factor of 10 decrease in quench rate is larger than $r_{c}^{-1} \sim 10^{5}$ and $r_{p}^{-1}>10^{5}$ (Fig. 4(a)), and in part because for $t_{w} \gtrsim 10^{6.5}$ systems have crossed into the preterminal (logarithmic) relaxation regime at $t^{\prime}=t-t_{w}=0$. Both polymer and colloidal crystallites continue to slowly add contacts and close-packed monomers, and the $t_{w}$-dependence should not vanish until equilibrium values of $\left\langle N_{c}\right\rangle$ and $\left\langle N_{c p}\right\rangle$ are reached.

(iv): At large $t^{\prime}$, the adjacency matrix autocorrelation function decays logarithmically. The crossover to logarithmic decay of $P_{\mathrm{AMAC}}\left(t_{w}, t^{\prime}\right)$ corresponds to the pre-terminal regime of logarithmic growth of $\left\langle N_{c}\right\rangle$ and $\left\langle N_{c p}\right\rangle$ (Fig. 4(b) and (c)).

9 This could be examined quantitatively by excluding successively more distant chemical neighbors (e.g. 2nd nearest, 3rd nearest), and by considering only chemically interior sections of polymers. A detailed analysis is left for future studies of near-equilibrium and equilibrium systems.
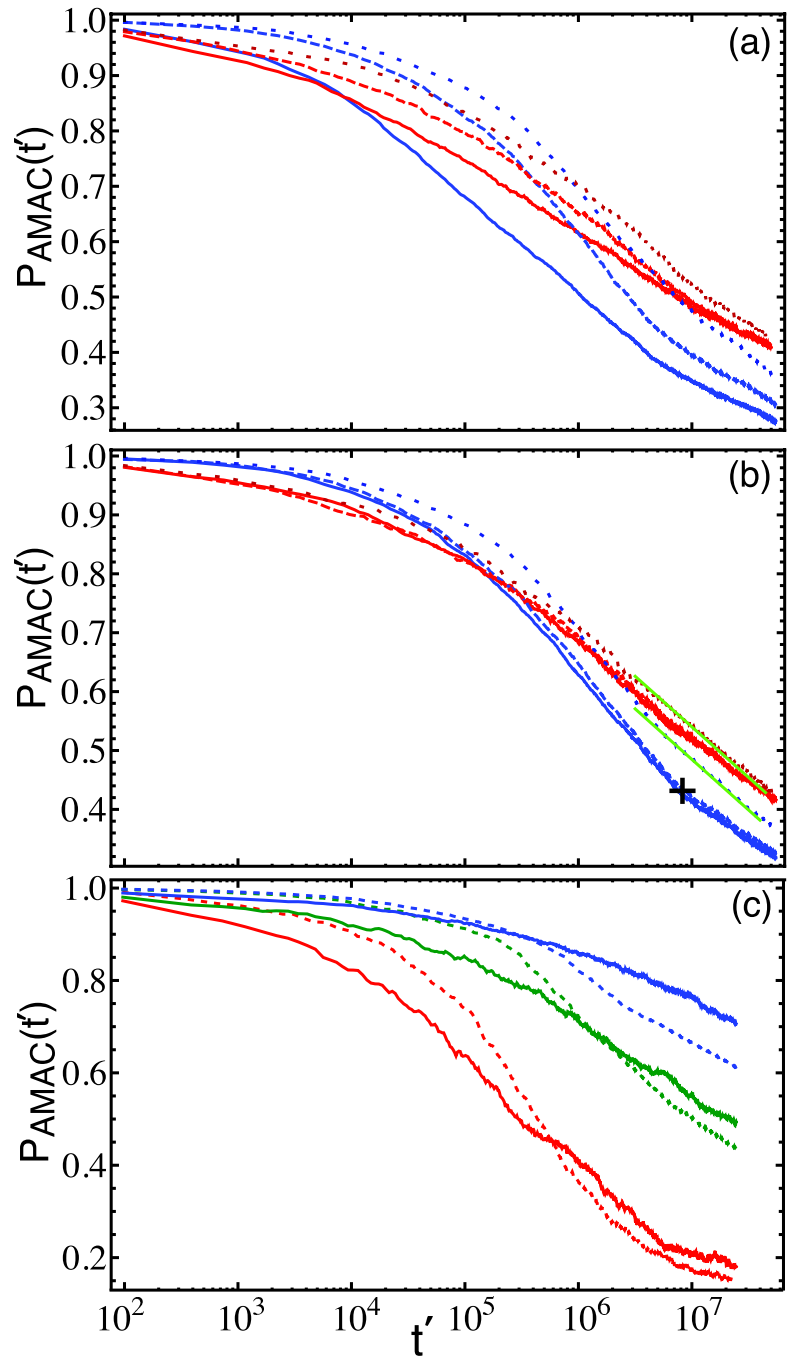

Fig. 6 The adjacency matrix autocorrelation function $P_{A M A C}\left(t_{w}, t^{\prime}\right)$ following (a) $|\dot{T}|=10^{-6}$ and (b) $10^{-7}$ thermal quenches. Protocols and systems for panels (a-b) are the same as in Fig. 4 Results for polymers (colloids) are shown in red (blue). $P_{A M A C}\left(t_{w}, t^{\prime}\right)$ is measured as a function of time $t^{\prime}$ after waiting times $t_{w}=0, t_{w}=10^{5.5}$, and $t_{w}=10^{6.5}$ (solid, dashed, and dotted curves, respectively). The solid green lines in (b) show fits to logarithmic behavior at long times for $t_{w}=10^{6.5}$ and the black $(+)$ sign denotes crossover into the terminal relaxation regime for colloids. Panel (c) shows how differences in $P_{A M A C}\left(t_{w}=0 ; t^{\prime}\right)$ between slowly quenched $\left(|\dot{T}|=2.5 \cdot 10^{-8}\right)$ polymeric and colloidal systems vary with $N$. Solid curves represent polymeric and dashed curves represent colloidal results, while colors indicate $N=40$ (red), $N=100$ (green), and $N=250$ (blue). Each curve in (c) represents an ensemble average over 40 statistically independent systems.

For colloids and small $t_{w}$, a decrease in the slope of this logarithmic decay corresponding to crossover into the terminal re- 
laxation regime is indicated by the + symbol in Fig. 6 (b). No such change in slope occurs for polymers over the same range of $t^{\prime}$. This is consistent with the idea that local relaxations in polymers are slower due to chain-connectivity constraints on rearrangements.

(v): Figure 6(c) illustrates the variation of $P_{\mathrm{AMAC}}\left(t_{w}=\right.$ $\left.0 ; t^{\prime}\right)$ with $N$ for slowly quenched systems. Characteristic contact decorrelation rates $s_{\text {cont }}$ decrease sharply with increasing $N$; for example, the low- $t^{\prime}$ plateau lengthens with increasing $N$, and the $t^{\prime}$ at which $P_{\text {AMAC }}=0.8$ is $2-3$ orders of magnitude greater for $N=250$ than for $N=40$. Since this $N$-dependent decrease in $s_{\text {cont }}$ is similar for polymeric and colloidal crystallites (which, as we have shown, possess similar structure), we attribute it to the increasing contribution of crystallite cores where reneighboring dynamics are slow because $\eta$ is high and particles are more sterically constrained. Similarities and differences between polymeric and colloidal results are consistent with those expected from (i). For all $N$, as in panels (a-b), polymers relax faster than colloids at low $t^{\prime}$ because $T_{f}=(7 / 8) T_{\text {cryst }}$ is higher, and slower at large $t^{\prime}$ because of topologically restricted rearrangement ( $c f$. Figs. 7 . 8.) Both the "crossover" $t$ ' at which $P_{\mathrm{AMAC}}^{\text {coll }}=P_{\mathrm{AMAC}}^{\text {pol }}$ and the ratio $P_{\mathrm{AMAC}}^{p o l} / P_{\mathrm{AMAC}}^{p o l}$ beyond this crossover time increase with increasing $N$.

In this paper we focus on the glassy dynamics of crystallite formation (where about half of the contacts existing at the termination of the quench have not been broken), not complete reorganization. Below, we show that there are significant differences between large-scale polymeric and colloidal rearrangements in this regime. In the remainder of this section, we will focus on $N=100$ collapsed states generated using protocol 2 with thermal quench rate $|\dot{T}|=10^{-7}$.

Statistical comparison of rearrangements in polymeric and colloidal crystallites: We describe rearrangement events using the two-dimensional parameter space $\left(N_{c}, N_{c p}\right)$, where tangent-sticky-sphere polymers and colloids have the same inherent structures. $\frac{42}{2}$ In Fig. 7 we show the probability distribution $P\left(\Delta N_{c}, \Delta N_{c p}\right)$ for crystallite rearrangements to add $\Delta N_{c}$ contacts and $\Delta N_{c p}$ close-packed particles in crystallites over time intervals $\Delta t=10^{3} \Pi$ Results are calculated for the range $10^{6.5} \leq t \leq 10^{7}$ where both colloids and polymers are in the preterminal relaxation regime. $P\left(\Delta N_{c}, \Delta N_{c p}\right)$ is proportional to the integrated rate

$$
R\left(\Delta N_{c}, \Delta N_{c p}\right)=\iint s\left(\Delta N_{c}, \Delta N_{c p}, N_{c}^{0}, N_{c p}^{0}\right) d N_{c}^{0} d N_{c p}^{0}
$$

for all transitions that add $\Delta N_{c}$ contacts and $\Delta N_{c p}$ closepacked particles in crystallites originally posessing $N_{c}^{0}$ con-

\| Note that for the physically reasonable values $m=10^{-24} \mathrm{~kg}, D=1 \mathrm{~nm}$, and $\epsilon \simeq 10 k_{B} T$ at room temperature, $\tau \simeq 5 \mathrm{ps}$, and timescales $\sim \Delta t$ can be probed by neutron spin echo experiments, e.g. for the purpose of characterizing protein dynamics. $\underline{\underline{43}}$ tacts and $N_{c p}^{0}$ close-packed particles, i.e. $R\left(\Delta N_{c}, \Delta N_{c p}\right) \simeq$ $P\left(\Delta N_{c}, \Delta N_{c p}\right) / \Delta t$. This becomes exact in the limit $R\left(\Delta N_{c}, \Delta N_{c p}\right) \Delta t \rightarrow 0$. Thus, the data in Fig. 7 provides a basis for comparing free energy barriers and transition rates in these systems.

Figure 7 and Table 3, which characterize the shape of the distribution $P\left(\Delta N_{c}, \Delta N_{c p}\right)$, illustrate the dramatic differences between polymeric and colloidal rearrangements during logarithmic relaxation. Polymeric rearrangements are significantly more correlated than those for colloids. For example, the correlation coefficient

$$
c=\frac{\left\langle\left(\Delta N_{c}-\left\langle\Delta N_{c}\right\rangle\right)\left(\Delta N_{c p}-\left\langle\Delta N_{c p}\right\rangle\right)\right\rangle}{\sqrt{\left\langle\left(\Delta N_{c}-\left\langle\Delta N_{c}\right\rangle\right)^{2}\left(\Delta N_{c p}-\left\langle\Delta N_{c p}\right\rangle\right)^{2}\right\rangle}},
$$

where the averages are taken over all rearrangements, is larger by a factor of 2.5 and the cross correlation $\left\langle\Delta N_{c} \Delta N_{c p}\right\rangle$ is larger by a factor of 7.4 for polymers compared to colloids. The second moments of $P\left(\Delta N_{c}, \Delta N_{c p}\right),\left\langle\left(\Delta N_{c}\right)^{2}\right\rangle$ and $\left\langle\left(\Delta N_{c p}\right)^{2}\right\rangle$, are also larger for polymers than colloids.

Table 3 Statistical analysis of the data presented in Fig. 7 The top two rows are calculated by fitting to data in Fig. 4 in the preterminal regime $\left(10^{6.5} \leq t \leq 10^{7}\right)$ The middle column shows data for colloidal rearrangements excluding "floaters".

\begin{tabular}{lccc}
\hline Quantity & Colloids & Colloids (NF) & Polymers \\
\hline$\partial\left\langle N_{c}\right\rangle / \partial \log _{10} t$ & 6.3 & 6.3 & 1.4 \\
$\partial\left\langle N_{c p}\right\rangle / \partial \log _{10} t$ & 2.1 & 2.1 & 0.7 \\
$\left\langle\Delta N_{c}^{2}\right\rangle$ & 9.1 & 2.2 & 23 \\
$\left\langle\Delta N_{c p}^{2}\right\rangle$ & 0.41 & 0.32 & 3.2 \\
$\left\langle\Delta N_{c} \Delta N_{c p}\right\rangle$ & 0.66 & 0.39 & 4.9 \\
$c$ & 0.23 & 0.34 & 0.58 \\
\hline
\end{tabular}

An interesting feature of $P\left(\Delta N_{c}, \Delta N_{c p}\right)$ is that it is nonzero in quadrants II and IV ( $i$. e. negative $\Delta N_{c} \Delta N_{c p}$ ). Rearrangements occur in which the number of close-packed monomers decreases but the number of contacts increases overall, and vice versa. These are less likely for polymers than for colloids since covalent bonds impart greater cooperativity to rearrangements in polymers. $P\left(\Delta N_{c}, \Delta N_{c p}\right)$ is reasonably well fit by a $2 \mathrm{D}$ Gaussian functional form

$$
P^{*}\left(\Delta N_{c}, \Delta N_{c p}\right) \propto e^{-E\left(\Delta N_{c}\right)^{2}+F \Delta N_{c} \Delta N_{c p}-G\left(\Delta N_{c p}\right)^{2}},
$$

where $E$ is similar for polymers and colloids, $F$ is larger for polymers, $G$ is larger for colloids, and $-F /(2 E G)$ is larger for polymers. These results are consistent with our finding that $c$ is larger for polymers than for colloids.

The fit $P^{*}\left(\Delta N_{c}, \Delta N_{c p}\right)$ does, however, fail to capture several key features of rearrangements. In particular, $P\left(\Delta N_{c}, \Delta N_{c p}\right)$ has a sharper peak at the origin and "fat" (wider than Gaussian) tails. The fat tails of $P\left(\Delta N_{c}, \Delta N_{c p}\right)$ 

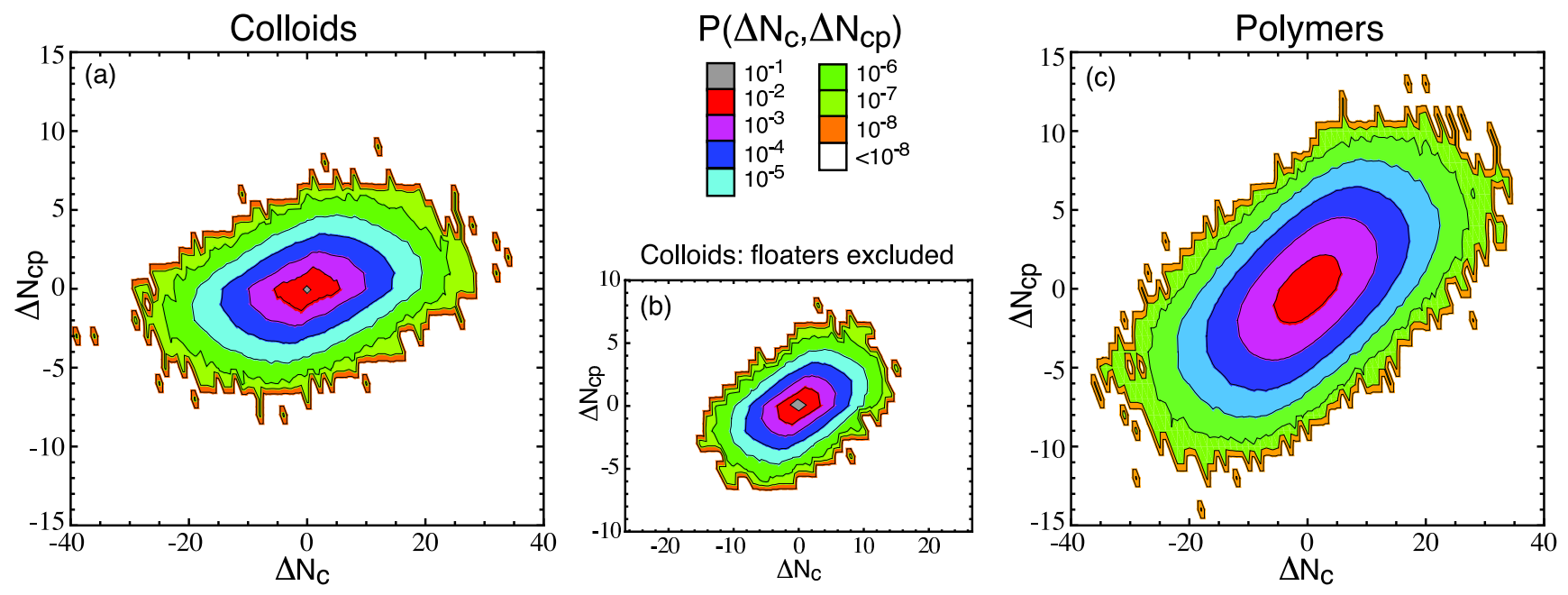

Fig. 7 Probability distributions $P\left(\Delta N_{c}, \Delta N_{c p}\right)$ for changes in the number of contacts and number of close-packed monomers over time intervals of $\Delta t=10^{3}$ following $|\dot{T}|=10^{-7}$ thermal quenches from $T_{i}>T_{\text {cryst }}$ to $T_{f} / T_{\text {cryst }}=7 / 8$ for (a) colloids, (b) colloids with "floaters" excluded, and (c) polymers. The different colored regions indicate bins in probability that differ by factors of 10 . Results are for the same systems analyzed in Figs. 4 and 6 b). To capture the "pre-terminal" logarithmic relaxation regime, only results for $10^{6.5} \leq t \leq 10^{7}$ are presented.

are dominated by events where a monomer escapes from or rejoins the crystallite (for colloids), or a chain end becomes "floppy" (for polymers). These events tend to be associated with rearrangements with large $\Delta N_{c}$ and small $\Delta N_{c p}$ for colloids, and large $\Delta N_{c}$ and $\Delta N_{c p}$ for polymers (the difference being that covalent bonds impart a large degree of coupling between the exterior and close-packed interior of crystallites). While rare, these events may dominate large rearrangements in which many changes of contacts occur.

To quantify this effect, we analyze colloidal rearrangements with "floaters" excluded, i.e. where all particles possess at least one interparticle contact both before and after the rearrangement. Since monomers in polymers always have at least one contact, excluding floaters provides a more direct method to quantify the role of topology in controlling the rearrangements occurring during slow crystallite growth. Figure $7 \mathrm{~b}$ ) and the "Colloids (NF)" data in Table 3 present this analysis. The "no-floater" subset of colloidal rearrangements possesses significantly smaller fluctuations (in terms of $\left\langle\Delta N_{c}^{2}\right\rangle$ and $\left\langle\Delta N_{c p}^{2}\right\rangle$ ) and is more correlated (in terms of $\left\langle\Delta N_{c} \Delta N_{c p}\right\rangle$ ). However, no-floater colloidal rearrangements remain significantly less correlated than those for polymers. Thus we claim that the distributions $P\left(\Delta N_{c}, \Delta N_{c p}\right)$ differ dramatically for polymers and colloids (i.e. are more correlated for polymers) precisely because their free energy landscapes are different, which in turn is a direct consequence of the presence or lack of covalent bonds.

The large width of the distribution $P\left(\Delta N_{c}, \Delta N_{c p}\right)$ implies that the dynamics of crystallite growth in the pre-terminal re- laxation regime are highly heterogeneous, especially for polymers. One source of dynamical heterogeneity, as suggested by Fig. 7 is surface effects. Rearrangements at the surfaces of crystallites (where monomers possess low $\eta$ ) are qualitatively different than those occurring within the close-packed cores. To illustrate the role of surface effects and covalent bonds, we now compare typical rearrangement events characteristic of those occurring in $(N=100)$ polymeric and colloidal crystallites.

Visualization of typical rearrangements in polymeric and colloidal crystallites: Figure 8 shows a colloidal rearrangement with $\Delta N_{c}=8, \Delta N_{c p}=2((\mathrm{a})$ and (b)) and a polymeric rearrangement with $\Delta N_{c}=7, \Delta N_{c p}=4((\mathrm{c})$ and (d)). These are roughly equiprobable, with $\log _{10} P\left(\Delta N_{c}, \Delta N_{c p}\right) \approx$ -3.5 , and the ratio $\Delta N_{c p} / \Delta N_{c} \approx c$. (See Table 3) Before the colloidal rearrangement, the red monomer has only one contact; during the rearrangement, it settles into a groove on the surface of the crystallite and adds three new contacts. At the same time, the right hand side of the crystallite undergoes a large rearrangement, which improves its stacking order, and five other contacts and two other close-packed atoms are added elsewhere. It is likely that the initial "looseness" of the red monomer destabilizes the crystallite and gives rise to the soft mode that causes this large rearrangement.

For polymers, the picture is qualitatively similar, but is further complicated by the fact that the interior and exterior of crystallites are topologically connected. Large rearrangements are often initiated when a chain end is at the surface and relatively loose. Note that in Fig. 8 (c) and (d) the color varies 

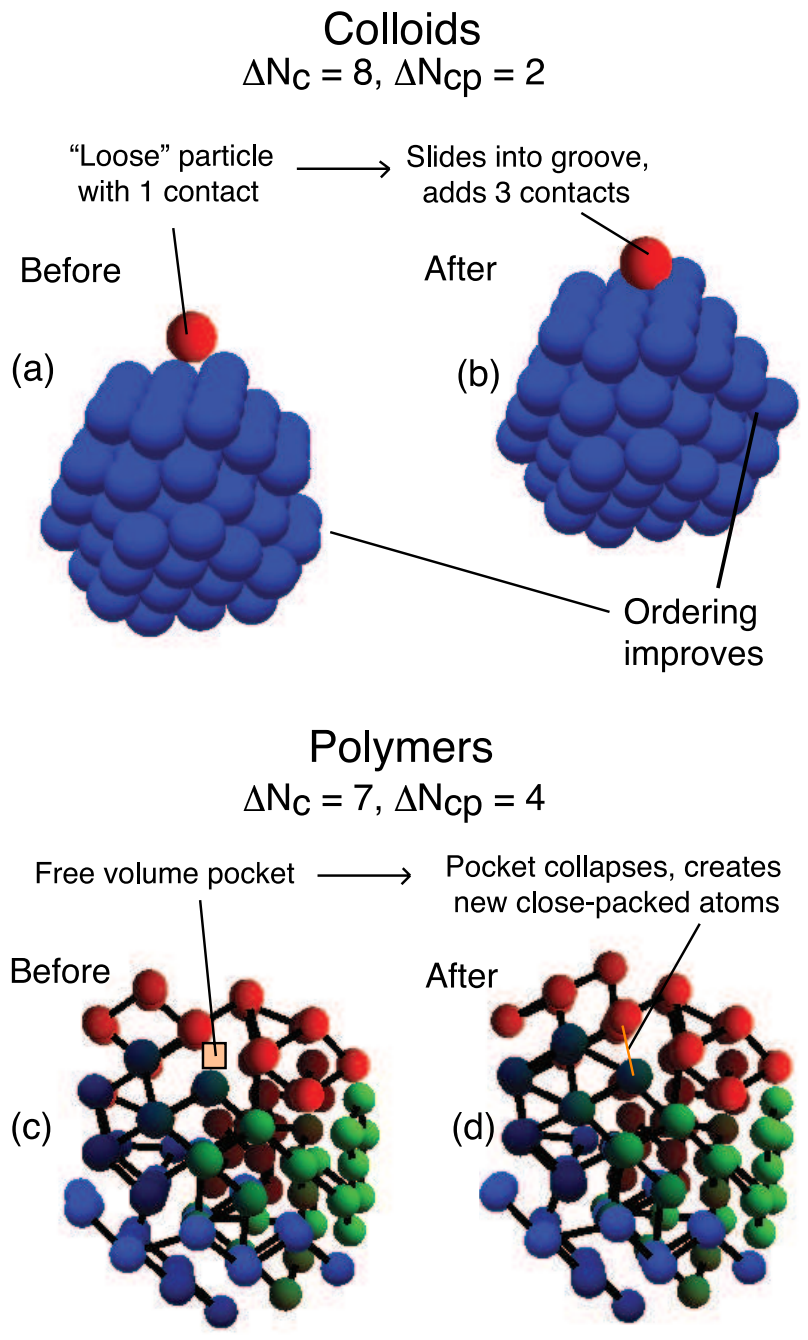

Polymers

$$
\Delta N_{C}=7, \Delta N_{C p}=4
$$

Pocket collapses, creates new close-packed atoms

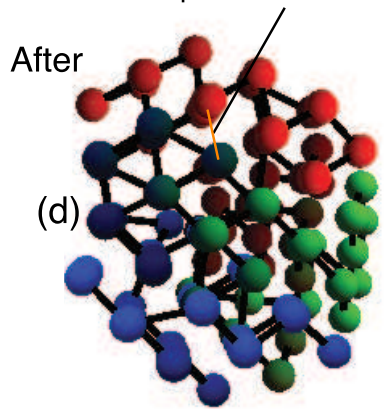

Fig. 8 Visualizations of typical moderately-sized rearrangement events for polymeric and colloidal crystallites. Panels (a) and (b) show the colloidal crystallite before and after a colloidal rearrangement event with $\Delta N_{c}=8$ and $\Delta N_{c p}=2$ and (c) and (d) show the polymeric crystallite before and after a polymeric rearrangement event with $\Delta N_{c}=7$ and $\Delta N_{c p}=4$. The orange color shading in (c) and line in (d) show where new close-packed particles are added.

from red to green to blue as the monomer index increases from 1 to 100 . Before the rearrangement, the chain end including monomer 100 is relatively loose (with only 3 contacts), and a pocket exists in the crystallite with above average free volume. During the rearrangement a segment of the polymer including this end executes a "flip" that collapses the pocket. The chain end monomer adds three contacts, and four other contacts and close-packed atoms are added elsewhere.

Covalent bonds suppress large rearrangements less when chain ends exist on the exterior of crystallites. In the rearrangement event depicted in Fig. 8 (c) and (d), the path of the covalent backbone through the crystallite is not particularly tortuous - it proceeds in a relatively orderly fashion from upper-right to lower-left. Entropic factors such as "blocking" suppress the probability for chain ends to exist in the interior, $\stackrel{21,39}{ }$ otherwise large rearrangements would be even further suppressed.

In summary, despite qualitative similarities, polymer topology produces the large quantitative differences in slow crystallite growth and rearrangements illustrated above in Figs. 48 and Table 3 Even larger and rarer polymeric rearrangements than those depicted in Fig. 8 involve cooperative rearrangements of sub-chains that do not include chain ends. Such large-scale rearrangements occur within the interior of the crystallite; their initiation requires a large (negative) $\Delta N_{c}$ and $\Delta N_{c p}$, and hence they possess large activation energies. These are the slowest relaxation mechanisms, and it is likely that they control the approach to the ergodic limit in polymer crystallites.

\section{Discussion and Conclusions}

In this article, we compared the crystallization dynamics of single-chain polymers and colloids. The use of model systems with hard-core-like repulsive and short-range attractive interparticle potentials yielded contact-dominated crystallization and allowed us to characterize crystalline order via measures such as the number of contacts $N_{c}$, the number of close-packed particles $N_{c p}$, and the contact degree distribution $P(\eta)$. Our use of a model in which covalent and noncovalent bonds have the same equilibrium bond length yielded the same low energy structures for polymeric and colloidal systems, allowing us to isolate the role of chain topology on the dynamics of crystallite formation and growth.

Particular attention was paid to the effect of thermal quench rate. Slow thermal quench rates yield first-order like transitions to crystallites at $T=T_{\text {cryst }}$. The ratio of $T_{\text {cryst }}$ for polymers to that for colloids can be obtained roughly by counting degrees of freedom. Comparison of polymeric and colloidal crystals at equal values of $T / T_{\text {cryst }}$ showed they possess similar structure (i.e. $N_{c}, N_{c p}$, and $P(\eta)$ ), and thus occupy similar positions on their respective free energy landscapes, despite significantly different absolute $T$. Higher quench rates yielded rate-dependent effects and glassy relaxation from partially disordered to more ordered configurations. While the marked slowdown in dynamics at $T=T_{\text {cryst }}$ and consequent ratedependent glassy behavior for crystal-forming systems possessing phase diagrams like Fig. 22 a) is understood in terms of a crossover to potential energy landscape dominated dynamics 44 with decreasing temperature, the role of covalent backbones (and consequently, different energy landcapes) in producing the strongly differing nonequilibrium responses for polymers and colloids reported in this paper has not been pre- 
viously isolated. We showed that although polymer crystallites nucleate faster because of the cooperative dynamics imparted by their covalent backbones, chain connectivity slows their relaxation towards maximally ordered structures.

Crystallites can rearrange in many different ways (e.g. with different changes $\Delta N_{c}$ and $\Delta N_{c p}$ in the number of contacts and close-packed monomers, respectively). By measuring the transition probability $P\left(\Delta N_{c}, \Delta N_{c p}\right)$ in the regime where the degree of crystalline order exhibits slow logarithmic growth, we characterized how the rare collective rearrangement events which control the slow approach of crystallites to equilibrium are affected by polymer topology. Significant differences between $P\left(\Delta N_{c}, \Delta N_{c p}\right)$ in polymeric and colloidal crystallite formation are attributable to the increased cooperativity of rearrangements required by the covalent backbone.

Strong finite size effects have been observed in both equilibrium and nonequilibrium polymer-collapse studies. 2.17,45 Here we examined system size effects using measures of order such as $N_{c}$ and $N_{c p}$ that vary rapidly with the number of particles $N$ due to concomitant variation in the ratio of crystallite surface area to volume. This variation did not change any of the qualitative features reported above, and quantitative differences were as expected; dynamical slowdown of relaxation associated with restricted motion imposed by the covalent backbone strengthens with increasing $N$.

It is well known that bond-angle interactions play a significant role in controlling crystallization of most synthetic polymers. While this study considered flexible chains, it serves as a basis for future studies of more realistic models by elucidating the role polymer topology plays in controlling the glassy dynamics of crystallization. Our results may also be directly applicable to understanding the collapse behavior of flexible "colloidal polymers", which have recently been shown to selfassemble into tunable, compact nanostructures, $\underline{\underline{25}}$ as well as very flexible natural polymers such as single stranded DNA. $\underline{46}$ Future work will examine how crystallization and packing are affected by semiflexibility, as well as effects of topology on the dynamics of equilibrium crystallites.

\section{Acknowledegements}

All MD simulations were performed using the LAMMPS molecular dynamics simulation software. ${ }^{47}$ We thank Steven J. Plimpton for developing enhancements to LAMMPS for the long-time runs, S. S. Ashwin and K. Dalnoki-Veress for helpful discussions, and Adam Hopkins for providing the

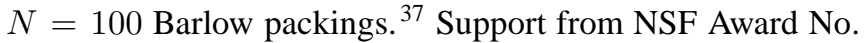
DMR-1006537 is gratefully acknowledged. This work also benefited from the facilities and staff of the Yale University Faculty of Arts and Sciences High Performance Computing Center and NSF grant No. CNS-0821132 that partially funded acquisition of the computational facilities.

\section{References}

1 M. P. Taylor, W. Paul and K. Binder, J. Chem. Phys., 2009, 131, 114907; M. P. Taylor, W. Paul and K. Binder, Phys. Rev. E, 2009, 79, 050801.

2 D. T. Seaton, T. Wüst and D. P. Landau, Phys. Rev. E, 2010, 81, 011802.

3 K. A. Dill and S. B. Ozkan and M. S. Shell and T. R. Weikl, Annu. Rev. Biophys. 37, 289 (2008); Y. Chen. et. al., Arch. Biochem. Biophys. 469, 4 (2008).

4 N. D. Socci and J. N. Onuchic, J. Chem. Phys., 1994, 101, 1519.

5 G. Foffi, E. Zaccarelli, F. Sciortino and P. Tartaglia, J. Stat. Phys., 2000, 100, 363.

6 T. Cellmer, D. Bratko, J. M. Prausnitz and H. W. Blanch, Trends Biotechnol., 2007, 25, 254.

7 M. Doi and S. F. Edwards, The Theory of Polymer Dynamics, Clarendon Press (Oxford), 1986.

8 E. A. Dimarzio, J. Polym. Sci. Part B - Polym. Phys., 2006, 44, 2612.

9 Previous studies $\frac{10}{}$ comparing collapsed states of polymers and colloids with system sizes comparable to those considered in this paper have employed much softer (LennardJones) interactions for which covalent bond stretching can produce greater differences in the low energy states, and have focused on equilibrium properties.

10 S. Schnabel, M. Bachmann, and W. Janke, J. Chem. Phys., 2009, 131, 124904; F. Calvo, J. P. K. Doye, and D. J. Wales, J. Chem. Phys., 2002, 116, 2642.

11 S. Sastry, P. G. Debenedetti, S. Torquato and F. H. Stillinger, Nature, 1998, 393, 554.

12 L. Mandelkern, A. Prasad, R. G. Alamo and G. M. Stack, Macromolecules, 1990, 23, 3696.

13 Studies examining the effects of polymer topology must not allow crossing of covalent bonds. The lowest barrier $\Delta U_{\text {cross }}$ for covalent bond crossing can be estimated ${ }^{14}$ by placing two bonds on opposite corners of a square. For the harmonic spring potential (Eq. 1), $\Delta U_{\text {cross }}=k(6-$ $4 \sqrt{2}$ ). For the temperatures and spring constant $k=1600 \epsilon$ considered here, $\Delta U_{\text {cross }} \gtrsim 10^{3} k_{B} T$.

14 S. K. Sukumaran, G. S. Grest, K. Kremer and R. Everaers, J. Polym. Sci. Part B - Polym. Phys., 2005, 43, 917.

15 S. Chakrabarty and B. Bagchi, J. Chem. Phys, 2010, 133, 214901.

16 P. R. ten Wolde, M. J. Ruiz-Montero and D. Frenkel, J. Chem. Phys., 1996, 104, 9932.

17 M. V. Massa and K. Dalnoki-Veress, Phys. Rev. Lett. 92, 255509 (2004); M. V. Massa, J. L. Carvahlo and K. Dalnoki-Veress, Phys. Rev. Lett. 97, 247802 (2006); J. L. Carvahlo and K. Dalnoki-Veress, Phys. Rev. Lett. 105, 237801 (2010). 
18 The small $r_{c}$ suppresses local icosohedral order in favor of local fcc/hcp order; this is likely one of the reasons these systems exhibit direct "gas"-solid transitions.

19 T. Biedl et. al., Discrete Comput. Geom., 2001, 26, 269.

20 N. Arkus, V. N. Manoharan and M. P. Brenner, Phys. Rev. Lett., 2009, 103, 118303.

21 R. S. Hoy and C. S. O'Hern, Phys. Rev. Lett., 2010, 105, 068001.

22 Results in the sticky-sphere $(k \rightarrow \infty)$ limit are known to provide a reference for perturbative studies of other forms of $U(r)$ with hard-core-like repulsions and differently ranged attractions..$^{5,23}$ Eq. 1 is also a first-order approximation for the depletion interactions typical of nano-selfassembling colloidal and "colloid-polymer" systems. ${ }^{24,25}$

23 T. W. Cochran and Y. C. Chiew, J. Chem. Phys., 2006, 124, 224901.

24 G. Meng, N. Arkus, M. P. Brenner and V. N. Manoharan, Science, 2010, 327, 560.

25 S. Sacanna, W. T. M. Irvine, P. M. Chaikin and D. J. Pine, Nature, 2010, 464, 575.

26 F. R. K. Chung, Spectral Graph Theory, American Mathematical Society, 1996.

27 W. Kob, J.-L. Barrat, F. Sciortino and P. Tartaglia, J. Phys. Cond. Matt., 2000, 12, 6385.

28 A. M. Puertas, M. Fuchs and M. E. Cates, Phys. Rev. Lett., 2002, 88, 098301.

29 M. H. J. Hagen and D. Frenkel, J. Chem. Phys., 1994, 101, 4093.

30 The curvature in $E_{\text {pot }}(T)$ for polymers indicates the well understood progression from extremely good solvent behavior at high $T$ to "poor" (but, for this potential, not poor enough to induce collapse into globules) solvent behavior at $T$ just above $T_{\text {cryst }}$.

31 This argument should become more accurate in the limit of vanishing attraction range; see e. g. M. P. Taylor, J. Chem. Phys., 2003, 118, 883.

32 S. Alexander, Phys. Rep., 1998, 296, 65.

33 K. A. Dill and H. S. Chan, Nature Struct. Bio., 1997, 10.

34 N. Arkus, V. N. Manoharan and M. P. Brenner, Discrete and Comput. Geom., to be published..

35 A. V. Tkachenko and T. A. Witten, Phys. Rev. E, 1999, 60, 687.

36 A. Widmer-Cooper, H. Perry, P. Harrowell and D. R. Reichman, Nature Physics, 2008, 4, 711.

37 A. B. Hopkins, F. H. Stillinger and S. Torquato, Phys. Rev. E, 2011, 83, 011304.

38 W. Barlow, Nature, 1883, 29, 186.

39 N. C. Karayiannis, K. Foteinopoulou and M. Laso, Phys. Rev. E, 2009, 80, 011307.

40 N. C. Karayiannis, K. Foteinopoulou and M. Laso, Phys.
Rev. Lett., 2009, 103, 045703.

41 N. C. Karayiannis, K. Foteinopoulou and M. Laso, J. Chem. Phys., 2009, 130, 164908; M. Laso, N. C. Karayiannis, K. Foteinopoulou, M. L. Mansfield and M. Kröger, Soft Matter, 2009, 5, 1762.

42 F. H. Stillinger, Science, 1995, 267, 1935.

43 R. Biehl, M. Monkenbush and D. Richter, Soft Matter, 2011, 7, 1299.

44 T. B. Schrøder, S. Sastry, J. C. Dyre and S. C. Glotzer, J. Chem. Phys., 2000, 112, 9834.

45 L. M. Lopatina, C. J. O. Reichhardt and C. Reichhardt, Phys. Rev. E, 2011, 84, 011303.

46 F. Latinwo and C. M. Schroder, Soft Matter, 2011, 7, 7907.

47 S. Plimpton, J. Comp. Phys., 1995, 117, 1.

This journal is @ The Royal Society of Chemistry [year]

Journal Name, 2010, [vol], $1-13$ | 13 